\title{
Análise bibliométrica acerca da transmissão vertical do HIV
}

\author{
Bibliometric analysis about vertical HIV transmission
}

Análisis bibliométrico sobre la transmission vertical del VIH

Daniela de Aquino Freire ${ }^{1 *}$, Juliana da Rocha Cabral ${ }^{1}$, Marta Regina Chaves Camilo Fernandes ${ }^{1}$, Rebeca Coelho de Moura Angelim ${ }^{1}$, Martha Sthefanie Borba Costa ${ }^{1}$, Thais da Silva Oliveira ${ }^{1}$, Brígida Maria Gonçalves de Melo Brandão ${ }^{1}$, Regina Celia de Oliveira ${ }^{1}$, Inacia Sátiro Xavier de França ${ }^{1}$, Fatima Maria da Silva Abrão1.

\section{RESUMO}

Objetivo: Identificar o perfil bibliométrico da produção científica internacional sobre a transmissão vertical do HIV no campo da enfermagem. Métodos: A busca ocorreu na base de dados Scopus mediante utilização dos descritores oriundos do MeSH: "HIV"; "Infectious Disease Transmission Vertical"; "Nursing". Esse estudo analisou 36 artigos dos últimos 10 anos de publicação (2010-2020). Resultados: Todos artigos encontravamse na língua inglesa. Constatou-se um número elevado de publicação no ano de 2015, com um total de 08 artigos publicados. Houve um destaque de publicações nos periódicos Curationis e o African Journal of Primary Health Care and Family Medicine, as quais estão localizados no Continente Africano. Destacaram-se 17 autores como maiores produtores do assunto e a maioria deles são procedentes de instituições nos Estados Unidos da América. Os descritores mais comuns foram o "HIV", "Transmissão Vertical" e "Proteção e prevenção da transmissão vertical". Considerações Finais: A análise contribuiu para conhecer os periódicos que mais publicam na área, assim como os países que desenvolvem mais pesquisas voltadas ao tema e os autores que têm mais publicações e citações, além de Índice-H relevante. Os quais geram credibilidade internacional para os pesquisadores realizarem suas fundamentações e disseminações de informações relevantes em diversas áreas.

Palavras-chave: HIV, Transmissão vertical de doença infecciosa, Enfermagem.

\begin{abstract}
Objective: To identify the bibliometric profile of international scientific production on the vertical transmission of HIV in the field of nursing. Methods: The search took place in the Scopus database using the descriptors from the MeSH: "HIV"; "Infectious Disease Transmission Vertical"; "Nursing". This study analyzed 36 articles from the last 10 years of publication (2010-2020). Results: All articles were in the English language. There was a high number of publications in 2015, with a total of 08 articles published. There was a highlight of publications in the Curationis journals and the African Journal of Primary Health Care and Family Medicine, which are located on the African continent. 17 authors stood out as major producers on the subject and most of them come from institutions in the United States of America. The most common descriptors were "HIV", "Vertical Transmission" and "Protection and prevention of vertical transmission". Final Considerations: The analysis contributed to discover the journals that most publish in the area, as well as the countries that develop more research on the topic and the authors that have more publications and citations, in addition to the relevant $\mathrm{H}$-Index. Which generate international credibility for researchers to carry out their foundations and disseminate relevant information in several areas.
\end{abstract}

Key words: HIV, Infectious disease transmission, Vertical, Nursing.

${ }^{1}$ Universidade de Pernambuco (UPE), Recife - Pernambuco.

*E-mail: daniela_3439@hotmail.com 


\section{RESUMEN}

Objetivo: Identificar el perfil bibliométrico de la producción científica internacional sobre la transmisión vertical del VIH en el campo de la enfermería. Métodos: La búsqueda se realizó en la base de datos Scopus utilizando los descriptores del MeSH: "VIH"; "Transmisión de enfermedades infecciosas vertical"; "Enfermería". Este estudio analizó 36 artículos de los últimos 10 años de publicación (2010-2020). Resultados: Todos los artículos fueron en inglés. Hubo una gran cantidad de publicaciones en 2015, con un total de 08 artículos publicados. Se destacaron las publicaciones en las revistas Curationis y el African Journal of Primary Health Care and Family Medicine, que se encuentran en el continente africano. 17 autores se destacaron como grandes productores en el tema y la mayoría de ellos provienen de instituciones en los Estados Unidos de América. Los descriptores más comunes fueron "VIH", "Transmisión vertical" y "Protección y prevención de la transmisión vertical". Consideraciones finales: El análisis contribuyó a descubrir las revistas que publican más en el área, así como los países que desarrollan más investigación sobre el tema y los autores que tienen más publicaciones y citas, además de un índice $\mathrm{H}$ satisfactorio. Que generan credibilidad internacional para que los investigadores lleven a cabo sus fundamentos y difundan información relevante en varias áreas.

Palabras clave: VIH, Transmissión vertical de enfermedad infecciosa, Enfermería.

\section{INTRODUÇÃO}

O Vírus da Imunodeficiência Humana (HIV), responsável pela Síndrome da Imunodeficiência Adquirida (AIDS), representa um grande problema no contexto de saúde pública. Decorrido um período superior a 30 anos da descoberta do HIV esse ainda é considerado uma epidemia mundial e um dos mais sérios desafios mundiais, em caráter da sua gravidade (BRASIL, 2015; LACERDA JS, et al., 2019; UNITED NATIONS, 2016).

Inicialmente, acreditava-se, em relação às primeiras infecções pelo HIV, que estas estavam limitadas apenas a homossexuais, hemofílicos e usuários de drogas, que, naquela época, instituíram 'grupos de riscos'Alós AP (2018). Com o passar dos anos, essa descrição se modificou e a transmissão do vírus começou a disseminar com grande frequência em mulheres heterossexual menos favorecidas financeiramente e profissionais do sexo, demonstrando uma mudança no perfil da doença e caracterizando um aumento significativo na transmissão vertical (BRASIL, 2109).

No Brasil, desde 2000 até 2019, foram notificadas 125.144 gestantes infectadas com HIV. Considerando os dados absolutos de 2008 com os dados quantificados em 2018 houve um aumento de $36,7 \%$. Entretanto, de 2018 para 2019 foi registrado uma redução percentual de 50\% nas notificações. Em 2019, foram identificados 4.482 casos em gestantes (BRASIL, 2109).

O feto é exposto ao vírus durante a gestação, trabalho de parto, parto ou através do aleitamento materno. Por conseguinte, a transmissão vertical ocorre da mãe para o filho durante esse período. Dessa forma, a prevenção da transmissão vertical tem-se um desafio para os órgãos responsáveis pela prevenção e tratamento do HIV.

O Tratamento com Antirretrovirais na gestação é preconizado pelo Sistema Único de Saúde (SUS), com a adesão ao tratamento, a carga viral, consequentemente, será reduzida, o que favorece à prevenção da infecção do neonato (BRASIL, 2018).

A gestante HIV positivo, durante todo período do ciclo gravídico e puerperal, deve ser acompanhada por uma equipe multidisciplinar na atenção básica e Serviço de Atenção Especializada - SAE visando atender todas as suas necessidades. Dessa forma, a gestante terá uma melhor qualidade de vida e poderá diminuir a morbidade e mortalidade de mãe e filho (SOUZA CP, et al., 2016).

O processo de promoção, prevenção, detecção precoce e tratamento continuamente são princípios do cuidado às gestantes e puérperas com HIV. O olhar holístico, como o olhar clínico, é de extrema importância para que o profissional de enfermagem conceba vínculo com a gestante e possibilite a diminuição dos riscos de não adesão ao tratamento. Por tanto, acolher e cuidar de gestante posiciona o enfermeiro diante de 
implicações cruciais durante todo o período gestacional, dentre elas, a detecção precoce do HIV e a prevenção da transmissão vertical, através da quimioprofilaxia (LIMA SS, et al., 2017).

Mesmo com todo avanço tecnológico voltado ao HIV, ainda há um grande número de gestantes infectadas pelo HIV no mundo. Dessa maneira, surgiu a necessidade de aprofundar o conhecimento sobre o-HIV e sua transmissão vertical associado à prática de enfermagem, avaliando a produção científica acerca do tema. Consequentemente, justifica-se a relevância da análise bibliométrica como instrumento que permite obter informações dessas produções e proporcionando a construção de indicadores importantes para o meio social. De acordo com o exposto, objetivou-se identificar o perfil bibliométrico da produção científica mundial sobre o HIV e sua transmissão vertical no campo da enfermagem.

\section{MÉTODOS}

Trata-se de um estudo descritivo de análise bibliométrica. Essa forma de análise proporciona o entendimento para o desenvolvimento de um campo dentro do estado da arte, analisando as publicações referentes ao tema, aos autores mais citados, e, aos periódicos que mais publicam, podendo assim, facilitar o acesso às referências para pesquisadores (SOARES PB, et al., 2016; PELICIONI LCP, et al., 2018).

Essa facilidade do acesso foi possível a partir de 1960 com a automação dos dados, possibilitando com precisão análises quantitativas da produção científica, trazendo benefícios práticos e imediatos identificando tendências e crescimento do conhecimento em determinadas áreas (NASCIMENTO CC, 2014).

Em estudos de bibliografia estatística, Paul Otlet foi o primeiro a utilizar o termo bibliometria, segundo Nascimento CC (2014). A bibliometria, portanto, é definida como uma técnica que utiliza dados matemáticos e estatísticos para quantificar os índices de produção e disseminação do conhecimento científico (Araújo CA, 2006) e, possíbilita combinar o método quantitativo e qualitativo, auxiliando numa melhor tomada de decisão (HAYASHI MCPI, et al., 2007). A Bibliometria possui três leis básicas: Lei de Bradford (produtividade de periódicos), Lei de Lotka (produtividades de autores) e a Lei de Zipf (frequência de ocorrência de palavras).

Para a realização desse produto foram utilizadas as três leis básicas da bibliometria, introduzindo a seguinte pergunta norteadora: Qual a métrica da produção científica, na enfermagem mundial, a respeito da transmissão vertical do HIV? A coleta de dados realizou-se no período de Março de 2020.

A busca dos artigos ocorreu na base de dados SCOPUS mediante utilização dos descritores oriundos do MeSH: "HIV"; "Infectious Disease Transmission, Vertical"; "Nursing". Foi utilizado o operador booleano "AND". Os critérios de inclusão estabelecidos foram: Todos artigos disponíveis, sem restrição de idioma e no espaço temporal de 10 anos, período de 2010 - 2020.A base de dados Scopus é uma banco de dados de resumos e citações de artigos para jornais/revistas acadêmicos. Abrange cerca de 19,5 mil títulos de mais de 5.000 editoras internacionais, incluindo a cobertura de 16.500 revistas peer-reviewed nos campos científico, técnico, e de ciências médicas e sociais - Scorpus, (2014).

Realizou-se aí uma busca para fazer o levantamento referente à subárea de publicação, aos perfis de autores, que abrange o ano e número de publicações e de seus dados bibliográficos, referências e detalhes sobre o número de citações de cada documento publicado que ele tenha recebido - índice $\mathrm{H}$. Além disso, foi levantado os países e nome dos periódicos, modalidade do artigo, idioma e descritores.

\section{RESULTADOS E DISCUSSÃO}

Esse estudo buscou identificar o core lists, isto é, os periódicos mais produtivos, no espaço temporal previamente determinado, os autores que mais publicam na temática da transmissão vertical pelo HIV.e os descritores de maior frequência. Portanto, utilizando as três leis básicas da bibliometria. Foram encontrados 36 artigos que atendiam aos critérios de inclusão e aos objetivos da pesquisa, no processo de busca e seleção do material no banco de dados da SCOPUS. Do total de artigos, 16 tinham acesso aberto e em relação à subárea percebe-se que medicina foi a subárea que mais publicou (27 artigos) seguidos da Enfermagem ( 9 artigos), como será identificado na Tabela 1. 
Tabela 1 - Subárea dos artigos publicados entre 2010 e 2020. SCORPUS. Recife, PE, Brasil, 2020.

\begin{tabular}{cc}
\hline Subárea & No de artigos publicados \\
\hline Medicine Show preview for & 27 \\
Nursing Show preview for & 9 \\
Social Sciences Show preview for & 2 \\
Immunology and Microbiology Show preview for & 2 \\
Psychology Show preview for & 2 \\
\hline
\end{tabular}

Fonte: Freire DA, et al., 2020.

Quando se trata de idioma, após a análise dos resultados, é notório que todos os artigos científicos se encontram no idioma inglês. Esse achado pode estar relacionado ao interesse de pesquisadores em publicar artigos nesse idioma por ser o mais utilizado no meio científico.

Por conseguinte, transmite para a maior parte da população global informações que explorem assuntos tão pertinentes como o HIV e sua relação com a transmissão vertical. Com relação ao número de publicações por ano, 2010 a 2020, constatou-se um aumento no ano de 2015 com um total de 08 artigos publicados, conforme mostra a Tabela 2.

Tabela 2 - Distribuição dos artigos de acordo com o ano de publicação. SCOPUS, 2010-2020. Recife, PE, Brasil, 2020

\begin{tabular}{ccc}
\hline Ano de Publicação & N & $\%$ \\
\hline 2019 & 03 & $8,3 \%$ \\
2018 & 01 & $2,8 \%$ \\
2017 & 05 & $13,9 \%$ \\
2016 & 04 & $11,1 \%$ \\
2015 & 08 & $22,2 \%$ \\
2014 & 03 & $8,3 \%$ \\
2013 & 02 & $5,6 \%$ \\
2012 & 06 & $16,6 \%$ \\
2011 & 02 & $5,6 \%$ \\
2010 & 02 & $5,6 \%$ \\
Total & 36 & $100 \%$ \\
\hline
\end{tabular}

Fonte: Freire DA, et al., 2020.

Como mostra a tabela anterior, o aumento da quantidade de artigos publicados no ano de 2015 pode ser justificado pelo fato da UNAIDS, nesse mesmo ano, trazer dados de aumento de casos da AIDS no cenário mundial, tendo como principal foco países ocidentais.

O crescente número está associado ao acesso às medicações que são gratuitas no Brasil, favorecendo o tratamento. Dessa forma, ainda que em números gerais a epidemia tenha diminuição de casos, essa está associada a deficiência na aderência ao tratamento, tornando-se um grande desafio para ser controlada (UNADIS, 2015).

Em contrapartida, houve uma diminuição no número de publicações nos anos seguintes. Com isso, ressalta para a difícil tarefa na construção da produção científica, pois os pesquisadores podem enfrentar adversidades para divulgar suas produções, tendo em vista o rigor adotado pelos periódicos para aceite dos manuscritos, dentre outros fatores.

A Tabela 3 mostra os periódicos com maior número de artigos publicados acerca do tema e o estrato avaliado. 
Tabela 3 - Distribuição dos periódicos de acordo com o maior número de artigos publicados e estrato. SCOPUS, 2010-2020. Recife, PE, Brasil, 2020.

\begin{tabular}{ccc}
\hline Periódicos & No de artigos & *Estrato \\
\hline $\begin{array}{c}\text { Curationis } \\
\begin{array}{c}\text { African Journal of Primary Health } \\
\text { Care and Family Medicine }\end{array}\end{array}$ & 4 & $\mathrm{~B} 1$ \\
\hline $\begin{array}{c}\text { The Canadian nurse } \\
\text { Revista Latino-Americana de } \\
\text { Enfermagem }\end{array}$ & 2 & $\mathrm{~A} 2$ \\
\hline $\begin{array}{c}\text { International Journal of Infectious } \\
\text { Diseases }\end{array}$ & 2 & $\mathrm{~A} 2$ \\
\hline $\begin{array}{c}\text { Implementation Science } \\
\text { BMC Medical Ethics }\end{array}$ & 1 & $\mathrm{~B} 1$ \\
\hline Journal of Clinical Nursing & 1 & $\mathrm{~B} 1$ \\
\hline
\end{tabular}

Legenda: *Estrato segundo a Coordenação de Aperfeiçoamento de Pessoal de Nível Superior (CAPES), de 2016.

Fonte: Freire DA, et al., 2020.

A análise dos achados corrobora a Lei de Bradford (1934) - lei de dispersão do conhecimento, utilizada em periódicos para estimar seu grau de relevância em determinada área. Sendo assim, observou-se, em evidencia, a presença de dois periódicos com um maior número de publicações acerca da temática, como a Curationis e o African Journal of Primary Health Care and Family Medicine, as quais estão localizados no Continente Africano. Esse resultado evidencia conformidade com a lei de Bradford, também conhecida como de lei da dispersão e "permite", mediante a medição da produtividade das revistas, estabelecer núcleo e o as áreas de dispersão sobre um determinado assunto em um mesmo conjunto de revistas (VANTI NAP, 2002)

De acordo com a avaliação da CAPES, estes periódicos têm estrato B1. Apesar do estrato ser 'B', esses periódicos são de grande relevância por estarem inseridos nas maiores bases de dados do mundo e por se tratarem do HIV, um vírus que representa, em todas as regiões mundiais, um dos mais graves problemas de saúde, sendo intensificada no território africano por estar associado com problemas socioeconômicos, agravando a epidemia do HIV (UNAIDS, 2016)

Entende-se que esses países possuem um predomínio na taxa de HIV e transmissão vertical, necessitando da ampliação nos investimentos em serviços de saúde voltados para essas mulheres durante todo o pré-natal, parto e pós-parto, além da prevenção de agravos relacionados à prática sexual desprotegida, sendo necessário à distribuição de preservativos e medicamentos antirretrovirais.

Após o levantamento das informações correspondentes a cada autor, foi possível constatar que 17 autores se destacaram com o maior número de artigos publicados sobre o tema, publicando entre 91 e 603 artigos cada autor, e, possuindo um número importante de citações em documentos, conforme se observa na Tabela 4. Esse resultado corrobora a Lei de Lotka que defende o ideal de que quanto mais se publica, existe uma tendência de facilitar as publicações de um novo trabalho. Além disso, os pesquisadores que publicam resultados mais interessantes ganham mais reconhecimento (MALTRÁS BB, 2003). 
Tabela 4 - Distribuição dos 17 autores mais produtivos, conforme número de artigos publicados, Índice-H, país e citações. SCOPUS, 2010-2020. Recife, PE, Brasil, 2020

\begin{tabular}{|c|c|c|c|c|c|}
\hline № de Ordem & Autor & № de artigos & Índice H & País & № de citações \\
\hline 1 & Sabin CA & 603 & 89 & Reino Unido & 32.158 \\
\hline 2 & $\begin{array}{c}\text { Van } \\
\text { IJzendoorn MH }\end{array}$ & 537 & 91 & Holanda & 36.366 \\
\hline 3 & $\begin{array}{c}\text { Bärnighausen } \\
T\end{array}$ & 413 & 63 & Alemanha & 23.185 \\
\hline 4 & $\begin{array}{c}\text { Bakermans- } \\
\text { Kranenburg } \\
\text { MJ. }\end{array}$ & 381 & 73 & Holanda & 23.837 \\
\hline 5 & Yogey RD. & 375 & 56 & EUA & 11.586 \\
\hline 6 & Vãlimäki M & 230 & 32 & Hong Kong & 3.692 \\
\hline 7 & Suominen MT & 165 & 24 & Finlândia & 1.897 \\
\hline 8 & Manuel O & 149 & 28 & Suíça & 3.431 \\
\hline 9 & Tookey PA & 141 & 33 & Reino Unido & 4.401 \\
\hline 10 & Brysiewicz P & 125 & 14 & África do Sul & 760 \\
\hline 11 & $\begin{array}{c}\text { Van } \mathrm{R} \text { e Koen } \\
\mathrm{KA}\end{array}$ & 119 & 36 & EUA & 3.300 \\
\hline 12 & Malkin RA & 106 & 15 & EUA & 1.032 \\
\hline 13 & Juffer F & 103 & 42 & Holanda & 6.506 \\
\hline 14 & Rasch V & 97 & 20 & Dinamarca & 1.611 \\
\hline 15 & Ehlers VJD. & 94 & 11 & África do Sul & 322 \\
\hline $16^{*}$ & $\begin{array}{c}\text { Galvão, } \\
\text { Gimeniz MT }\end{array}$ & 91 & 10 & Brasil & 442 \\
\hline $16^{*}$ & Goon DT & 91 & 12 & África do Sul & 74 \\
\hline
\end{tabular}

Fonte: Freire DA, et al., 2020. *Mesmo número de ordem por apresentarem mesmo quantitativo de publicações.

Em relação ao país de origem, quatro autores são procedentes de instituições localizadas nos Estados Unidos da América, três da África do Sul e dois da Holanda, como mostra a Tabela 4.

Os países europeus, Reino Unido, Holanda e Alemanha, que apresentaram os maiores resultados em publicações sobre a temática pode ser justificada pela proximidade geográfica ao Continente Africano que é considerado como o marco inicial da epidemia de casos da AIDS.

Esses países destacam-se também com o índice h (número de artigos com citações maiores ou iguais a esse número) dos autores com grande impacto no que concerne aos seus artigos mais citados.

Vale ressaltar a preocupação da comunidade científica europeia em relação ao HIV, a qual realizou uma pesquisa sobre o cuidado contínuo relacionados ao HIV em 55 países da Europa e Ásia Central.

Os resultados mostraram que para melhor informar os programas de teste e tratamento do HIV, são necessárias abordagens e definições padrão de coleta de dados em todo atendimento ao HIV e que se os países desejam garantir um atendimento contínuo ao HIV, as pessoas que vivem com vírus precisam ser diagnosticadas prontamente, e o tratamento deve ser oferecido a todos (DREW RS, et al., 2017).

Com relação aos descritores, foi trabalhada a Lei de Zipf, também conhecida como Lei do Menor Esforço, que incide na medição de frequência do aparecimento das palavras em vários textos. Assim, é gerada uma lista ordenada de termos de uma determinada disciplina ou assunto (VANTI NAP, 2002).

Dos 36 artigos selecionados, 7 artigos não os apresentaram descritores. Dos que mencionaram descritores e/ou palavras-chave, contabilizou-se um total de 81 , os quais os mais prevalentes foram distribuídos de acordo com as principais temáticas estudadas pelos autores nas produções, como exposto na Tabela 5. 
Tabela 5 - Distribuição dos principais descritores utilizados pelos autores. Recife, PE, Brasil, 2020.

\begin{tabular}{ccc}
\hline Descritores & No de artigos & $\%$ \\
\hline HIV & 22 & $61,1 \%$ \\
Transmissão Vertical & 12 & $33,3 \%$ \\
Prevenção e controle do HIV & 05 & $13,9 \%$ \\
Enfermagem & 06 & $16,7 \%$ \\
Terapia antirretroviral & 06 & $16,7 \%$ \\
Gestação e HIV & 03 & $8,3 \%$ \\
Crianças com AIDS & 03 & $8,3 \%$ \\
Amamentação & 02 & $5,5 \%$ \\
\hline
\end{tabular}

Fonte: Freire DA, et al., 2020.

Vale salientar que o HIV é, ainda, uma pandemia e se constitui um problema de saúde pública, tornando a assistência à saúde um desafio mundial - Pires CP e Santos C (2018). Observa-se que o principal descritor presente neste estudo é 'HIV' presente em 22 artigos, seguido por 'Transmissão Vertical' com 12 artigos que estão relacionados ao tema proposto na pesquisa. Por fim, em dois artigos, surgiu a palavra 'Amamentação' que está associado ao tema e traz repercussões em relação à amamentação em mulheres que apresentam o HIV. O descritor amamentação foi abordado em 5,55\% dos trabalhos analisados, revelando que 0 aleitamento materno em puérperas soropositivas representa risco para a transmissão do vírus.

Segundo a Organização Mundial da Saúde, o sucesso da substituição do leite materno para a alimentação artificial depende da utilização de forma láctea que seja acessível a todos, seja sustentável e segura condições ideias. ainda, ressalta que essa precisa estar disponível em quantidade e frequência ideias para o crescimento e desenvolvimento da criança (GREENE S, et al., 2015; WHO, 2105). Em detrimento ao aumento do número de casos de Aids em mulheres tem-se um aumento da Transmissão Vertical do HIV, fazendo-se necessário focalizar inúmeros esforços na tentativa de impedir ou diminuir de forma significativa este tipo de transmissão.

A taxa de transmissão vertical chega a porcentagem de 30\% quando não se tem nenhuma forma de intervenção por parte dos profissionais envolvidos no cuidado à mulher gestante, mas esse índice cai para $1 \%$ quando se adota medidas de prevenção (REDMOND AM e MCNAMARA F, 2015).

Inserido nesse contexto, as mulheres necessitam ter acesso às informações sobre o cuidado com sua saúde e com o processo gestacional a fim de garantir uma gravidez saudável e com menor risco à criança. Para as gestantes diagnosticadas com HIV, os profissionais de saúde precisam pensar na possibilidade de sentimento de rejeição, por fatores como medo, angústia, preconceito e até mesmo pela descrença da eficácia das medicações. Estimular a adesão à terapia antirretroviral é essencial nesse processo (SILVA JM e SILVA F, 2018). Nota-se a importância do diagnóstico o mais precoce possível para que o tratamento possibilite a eficácia, em relação à transmissão vertical.

Vê-se a importância da enfermagem, principalmente na prevenção de complicações, realizando educação em saúde acerca do processo terapêutico, esclarecendo as possíveis dúvidas da paciente, oferecendo orientações inerentes ao aleitamento materno, explicando à paciente as vantagens em relação ao controle da carga viral, além da necessidade de uma adesão ao tratamento de forma eficaz, para assim contribuir de forma positiva evitando assim, a transmissão vertical.

O enfermeiro além de estimular a adesão medicamentosa também deve atuar de modo que o uso dos antirretrovirais possa trazer uma sensação de conforto para aquelas gestantes que se sentem culpabilidade pela possibilidade de transmissão vertical do HIV ao filho (SILVA JM e SILVA F, 2018). Outro descritor abordado foi sobre a 'Transmissão Vertical', correspondendo a 33,3\% dos estudos. Desde o primeiro caso de transmissão vertical do HIV em 1983, vem ocorrendo melhorias relevantes, em relação à prevenção.

No fim dos anos 90, o teste de HIV passou a ser rotina no pré-natal e o uso de antirretrovirais vem mostrando sua eficácia. Sobre o descritor antirretroviral, em 2012, houve uma diminuição na cobertura dos fármacos antirretrovirais durante o período de amamentação (49\%) do que durante a gravidez (62\%) (MIRANDA AE, et al., 2016; LIMA S, et al., 2017). 
Segundo a UNAIDS, a única maneira de se alcançar a meta de eliminação da transmissão vertical é por meio de estratégias bem planejadas e fundamentadas pelos princípios dos direitos humanos, respeito mútuo e inclusão. As formas opressivas não somente violam os direitos humanos fundamentais, como também deteriorizam as expectativas quanto ao fim da epidemia da Aids. Essas estratégias coercivas acabam por afastar as pessoas que precisam de atendimento nos serviços (UNAIDS, 2015).

Em virtude disso, o enfermeiro, como parte integrante de uma equipe multiprofissional voltada à assistência à essas mulheres, tem a responsabilidade de contribuir para, tomadas de decisão eficazes para a prevenção da transmissão vertical do HIV, seja durante a gestação até o pós parto e amamentação. Visto que, uma estratégia de cuidado bem planejada contribui para o alcance da eliminação da transmissão vertical do HIV.

Mesmo com tudo isso, a falta de empatia por parte dos profissionais de saúde a respeito da realidade social e as dificuldades que as mulheres vivenciam, contribuem de forma negativa para a adesão ao prénatal. Estas situações cooperam para a desinformação e o preconceito, sendo perceptível as gestantes. $O$ sentimento de discriminação leva mulher a rejeitar o tratamento. Dessa forma, é propagada a deficiência na educação e promoção à saúde, assim como, a falta de humanização dos profissionais em serviços de referência em saúde destinados a essa população (LIMA SS, et al., 2017).

Assim, ao examinar as evidências da rede de apoio das mulheres, sobressai a importância dessa rede na adesão às medidas preventivas para evitar a transmissão vertical e a relevância do profissional da saúde em conhecer essa rede, no propósito de incluir em seu planejamento assistencial as demandas resultantes desse contexto. Além disso, esses profissionais precisam proporcionar subsídios, provindo das informações e do conhecimento compartilhado, tornando desta forma instrumentos de promoção da saúde das mulheres (SILVA SS, et al., 2015). Por fim, percebe-se também a necessidade de organização dos serviços de saúde a fim de realizar planejamentos e desemvolver ações que busquem melhoria da identificação e acompanhamento dessas gestantes soropositivas. Para isso, o enfermeiro, como profissional integrante da equipe, desemvolve um papel ímpar.

A análise bibliométrica, utilizada nesse estudo, mostrou ser uma importante ferramenta para a avaliação de periódicos científicos, tanto pelos seus benefícios práticos quanto pelos seus benefícios imediatos. Contudo, como todo método de avaliação, possui suas vantagens e desvantagens, e, por isso, não possui unanimidade na sua aprovação. Ferreira AGC (2010) afirma que a bibliometria é indiscutivelmente uma ferramenta indispensável para o conhecimento de determinadas comunidades científicas, identificando comportamentos e tendências e, também, a qualidade das publicações.

\section{CONSIDERAÇÕES FINAIS}

Diante das inúmeras possibilidades de pesquisa utilizando a análise bibliométrica, esse estudo revelou que os artigos recuperados na base de dados da Scopus, no espaço temporal estabelecido, corroboraram às três leis da bibliometria, ou seja, os periódicos que mais publicam na área, os países que desenvolvem mais pesquisas voltadas ao tema, os autores que têm mais publicações e citações, os descritores mais utilizados por esses autores, além de $\mathrm{H}$-index relevantes, os quais geram credibilidade internacional para os pesquisadores. Apesar de um número relativamente baixo de artigos encontrados, esses apresentam dados robustos e de excelência. Vale salientar que, o tema ainda não atingiu a sua maturação científica do ponto de vista quantitativo, sendo assim, precisa-se enfatizar a necessidade de pesquisas voltadas a temática em questão, principalmente na área de enfermagem, pois o HIV e a transmissão vertical não pode ser vista somente como uma doença física, mas responsável por grandes impactos emocionais ao paciente e aos seus familiares.

\section{REFERÊNCIAS}

1. ALÓS AP. Corpo infectado/Corpus infectado: aids, narrativas e metáforas oportunistas. Revista Estudos Feministas. 2018; 27(3): 1-11.

2. ARAÚJO CA. Bibliometria: evolução histórica e questões atuais. Revista Em Questão. 2006; 12(1): 11-32. 
3. BIBLIOTECA DA ESCOLA DE ENGENHARIA. Ufrgs (Org.). Scopus tutorial. Rio Grande do Sul. 2014. Disponível em: https://www.ufrgs.br/bibicbs/tutoriais/ Acesso em: 10 de mar de 2020.

4. BRASIL. Ministério da Saúde - Secretaria de Vigilância em Saúde, Departamento de DST, AIDS e Hepatites Virais. Boletim Epidemiológico AIDS e DST. Brasília, 2015; 100p.

5. BRASIL. Ministério da Saúde. Boletim Epidemiológico - HIV/AIDS. Secretaria de Vigilância de Saúde. departamento de DST, Aids e Hepatites Virais. Número Especial. Brasília, 2019; 72p.

6. BRASIL. Ministério da Saúde. Protocolo clínico e diretrizes terapêuticas para prevenção da transmissão vertical de hiv, sífilis e hepatites virais. Brasília: Ministério da Saúde, Secretaria de Vigilância em Saúde, Departamento de IST, Aids e Hepatites Virais. Brasília, 2018; 72p.

7. DREW RS, et. al. HIV continuum of care in Europe and Central Asia. HIV Medicine. 2017; 18: 490-499.

8. FERREIRA AGC. Bibliometria na avaliação de periódicos científicos. Revista de Ciência da Informação, 2010; 11(3).

9. GREENE S, et al. "Why Aren't You Breastfeeding?": how mothers living with HIV talk about infant feeding in a "Breast Is Best" world. Health Care Women Int. 2015; 36(8): 883-901.

10. HAYASHI MCPI, et al. Um estudo bibliométrico da produção científica sobre a educação jesuítica no Brasil colonial. Revista Biblios. 2007; 8 (27): 1-18.

11. JOINT UNITED NATIONS. Global report: UNAIDS report on the global AIDS epidemic: 2016. Geneve: United Nations, 2016; 16p.

12. LACERDA JS, et al. Evolução medicamentosa do HIV no Brasil desde o AZT até o coquetel disponibilizado pelo sistema único de saúde. Revista Brasileira Interdisciplinar de Saúde. 2019; 1(4): 83-91.

13. LIMA SS, et al. HIV na gestação: pré-natal, parto e puerpério. Ciência \& Saúde. 2017; 10(1): 56-61.

14. MALTRÁS BB. Indicadores de Producción. In: MALTRÁS BARBA, Bruno. Los Indicadores Bibliométricos: fundamentos y aplicación al análisis de la ciencia. Gijón, Ediciones Trea, 2003. (cap. 4), 288p.

15. MIRANDA A, et al. Avaliação da cascata de cuidado na prevenção da transmissão vertical do HIV no Brasil. Caderno Saúde Pública. 2016; 32(9): 1-9.

16. NASCIMENTO CC. Revistas científicas: a busca pela qualidade e indicadores bibliométricos. Dissertação (Mestrado em Comunicação) - Escola de Comunicações e Artes./Universidade de São Paulo, são Paulo, 2014; 196 p.

17. PELICIONI LCP, et al. Application of a bibliometric tool for studyng space technology trends. Journal of Aerospace Technology and Management (JATM). 2018; 10: 1-8.

18. PIRES CP, SANTOS C. Qualidade de vida do paciente frente ao diagnóstico de HIV-AIDS. Revista Congrega Urcamp. 2018; 684-93.

19. REDMOND AM, MCNAMARA F. O caminho para eliminação da transmissão vertical do HIV. Jornal de Pediatria. 2015; 91(6): 509-11.

20. SILVA JM, SILVA F. A enfermagem e a prevenção da transmissão vertical do HIV: uma revisão integrative. Revista Interd. 2018; 11(1): 95-104.

21. SOUZA CP, et al. Incidência de transmissão vertical do HIV entre gestantes soropositivas cadastradas em um serviço de referência regional. Revista Cuidado Fundam. 2016; 8(2): 54526-4537.

22. SOARES PB, et al. Análise bibliométrica da produção científica brasileira sobre Tecnologia de Construção e Edificações na base de dados Web of Science. Revista Ambiente Construído. 2016; 16(1): 175-185.

23. UNAIDS. Programa Conjunto das Nações Unidas sobre HIV/AIDS. $2015 . \quad$ Disponível: https://nacoesunidas.org/agencia/unaids/. Acesso em 10 de mar. 2020.

24. UNAIDS. UNAIDS saúda DDAHV pela realização da 1a reunião do GT de certificação da eliminação de transmissão vertical do HIV e da sífilis. 2016. Disponível em: https://unaids.org.br/2016/11/unaids-sauda-realizacao-gtcertificacao-eliminacao-transmissao-vertical-hiv-sifilis/. Acesso em: 10 de mar. 2020.

25. UNAIDS. 90-90-90 Uma meta ambiciosa de tratamento para contribuir para o fim da epidemia de AIDS. Geneva, Switzerland: WHO; 2015. 38p.

26. VANTI NAP. Da bibliometria à webometria: uma exploração conceitual dos mecanismos utilizados para medir o registro da informação e a difusão do conhecimento. Ciência da Informação. 2002; 31(2): 152-62.

27. WORLD HEALTH ORGANIZATION-WHO. Guideline Uptades on HIV and infant feeding, 2016: the duration of breastfeeding and support from health services to improve feeding practices among mothers living with HIV. Geneva, Switzerland: WHO; 2016, 68p.

28. SILVA SS, et al. Rede de apoio a mulheres com HIV na prevenção da transmissão vertical: revisão integrativa. Revista Min. Enferm. 2015; 19(2): 225-231. 